\title{
Statistical properties of energy levels of chaotic systems: Wigner or non-Wigner
}

\author{
Jakub Zakrzewski ${ }^{1,2}$, Karine Dupret ${ }^{1}$ and Dominique Delande ${ }^{1}$ \\ ${ }^{1}$ Laboratoire Kastler-Brossel, Tour 12, Etage 1, Universite Pierre et Marie Curie, \\ 4 Place Jussieu, 75005 Paris, FRANCE \\ ${ }^{2}$ Instytut Fizyki, Uniwersytet Jagielloński, ulica Reymonta 4, 30-059 Kraków, POLAND*.
}

(November 1, 2018)

\begin{abstract}
For systems whose classical dynamics is chaotic, it is generally believed that the local statistical properties of the quantum energy levels are well described by Random Matrix Theory. We present here two counterexamples - the hydrogen atom in a magnetic field and the quartic oscillator - which display nearest neighbor statistics strongly different from the usual Wigner distribution. We interpret the results with a simple model using a set of regular states coupled to a set of chaotic states modeled by a random matrix.
\end{abstract}

Typeset using REVTEX

*permanent address 
Since the pioneering work of Bohigas et al [1], it has been numerically checked on a wide variety of systems [2,3] that the local statistical properties of a quantum system whose classical dynamics is chaotic, are well described by Random Matrix Theory. Especially, the statistical distribution of energy spacing between consecutive levels - also called Nearest Neighbor Spacing (NNS) distribution - has been shown to be in excellent agreement with the spacing distribution between consecutive eigenvalues of random matrices. For spinless systems with time-reversal symmetry, the Hamiltonian is real in a suitable basis and the Gaussian Orthogonal Ensemble (GOE) of random matrices has to be used. The NNS distribution of this ensemble is very close to the Wigner surmise [2, 3]:

$$
P(s)=\frac{\pi s}{2} \exp \left(-\frac{\pi s^{2}}{4}\right),
$$

where $P(s) \mathrm{d} s$ measures the probability of having a spacing in the interval $[s, s+\mathrm{d} s]$. In order to have dimensionless quantities, $s$ is here measured in units of mean level spacing.

The NNS distribution of specific quantum chaotic systems generally follows the Wigner prediction for highly excited states, that is in the semiclassical limit, $\hbar \rightarrow 0$. In this paper, we study numerically two simple systems whose classical dynamics are chaotic, but whose NNS distributions significantly differ from the Wigner distribution. These systems have in common that, in some part of the classical phase space, an adiabatic separation of the motion along two coordinates is possible. These regions of phase space are nevertheless coupled to more irregular regions and the resulting global motion is classically strongly chaotic. The surprising result is that, even for very highly excited states, the approximate adiabaticity is not washed out in the NNS distribution. Although these examples are clearly not generic because of the partial adiabatic separation, they are of major practical importance. For example, a consequence of this study is that the statistical properties of energy levels of the hydrogen atom in a magnetic field do not obey the Random Matrix prediction in the vicinity of the ionization threshold (a region widely experimentally studied in the last few years [4]), even for highly excited Rydberg states.

The first system we consider is the hydrogen atom in a magnetic field (along the $z$ axis) 
whose hamiltonian is in atomic units (neglecting relativistic, spin, QED, finite mass of the nucleus effects):

$$
H=\frac{\mathbf{p}^{2}}{2}-\frac{1}{r}+\frac{\gamma^{2}}{8}\left(x^{2}+y^{2}\right)
$$

where $\gamma$ denotes the magnetic field in units of $2.35 \times 10^{5} \mathrm{~T}$. The paramagnetic term $\gamma L_{z} / 2\left(L_{z}\right.$, the angular momentum along the $z$ axis is a good quantum number, together with parity) which can exactly be taken into account is dropped here. The classical dynamics of this system depends only on the scaled energy, $\epsilon=E \gamma^{-2 / 3}$, and is almost fully chaotic for $\epsilon>-0.12$.

Various studies of NNS distribution [5,6] - on spectra obtained either at constant magnetic field or at constant scaled energy - are in good agreement with the Wigner prediction above $\epsilon=-0.12$. However, careful checks close to the classical ionization threshold, $E=0$, have not been performed. The results presented here have been obtained in this region for the $\left(L_{z}=0\right.$, odd parity) series of energy levels. Spectra have been generated at constant magnetic field by numerical diagonalization of the hamiltonian in a huge Sturmian basis [5].6] using the Lanczos algorithm [7] which allows us to compute at low cost the few levels of interest in a narrow energy band.

The value of $\gamma$ ranges from $9 \times 10^{-5}$ to $6.35 \times 10^{-4}$ and the matrix size is up to 77000 , from which about 50 energy levels are computed (ranging between the $70^{\text {th }}$ and the $380^{\text {th }}$ excited state, depending on $\gamma$ ). For each $\gamma$ value, the energy spectrum is unfolded to determine the average density of states and the NNS distribution further computed. Because we want to study levels in a narrow energy band, only 5 to 20 spacings were computed for each $\gamma$ value. 478 NNS distributions at various $\gamma$ values - sufficiently far one from the other to ensure that they are not correlated - are then collected to build the distributions plotted in Fig.1 and Fig.2.

For reasons to be explained below, the data are collected separately in regions of constant $E / \gamma$. For example, Fig. 1 displays the NNS distribution for energy levels such that $-0.4 \gamma<$ $E<-0.3 \gamma$ (inset) and the corresponding cumulative NNS distribution, $\int_{0}^{s} P(x) \mathrm{d} x$, together 
with the Wigner prediction. Clearly, there is a significant deviation; especially, there are no large spacings. Fig. 2 displays the same comparison, but for energy levels lying closer to the ionization threshold, such that $-0.2 \gamma<E<-0.1 \gamma$. The deviation from the Wigner surmise is here even more spectacular, with the absence of spacings larger than 1.7 mean spacing.

A key point is that the relevant parameter describing the NNS distribution is the ratio $E / \gamma$. In Fig. 2, we show two different stretches of $\gamma$ values. Within statistical errors, the two distributions coincide, although the average value of $\gamma$ changes by a factor 2.5. This indicates that the observed deviation persists in the semiclassical limit $\gamma \rightarrow 0, E \rightarrow 0, E / \gamma$ fixed.

The relevant parameter $E / \gamma$ is not the scaled energy, which indicates that the deviations observed here are of quantum origin. A careful study of the phase space structure allows one to understand the origin of the phenomenon. Indeed, the diamagnetic potential $\gamma^{2} \rho^{2} / 8$ confines the motion transverse to the magnetic field, but not in the magnetic field direction. Hence, just below the ionization threshold, the electron can explore the region around the $z$ axis very far from the nucleus. In the region $|z| \gg \rho$, the Coulomb potential $1 / \sqrt{\rho^{2}+z^{2}}$ is almost equal to $1 /|z|$, leading to the adiabatic-like separation [4]:

$$
H \simeq H_{\mathrm{sep}}=H_{z}+H_{\rho}=\frac{p_{z}^{2}}{2}-\frac{1}{|z|}+\frac{p_{\rho}^{2}}{2}+\frac{\gamma^{2} \rho^{2}}{8} .
$$

The spectrum of $H_{\mathrm{sep}}$ as a sum of the 1-dimensional Coulomb, $H_{z}$, and of the harmonic oscillator, $H_{\rho}$, hamiltonians is

$$
E\left(n_{z}, n_{\rho}\right)=\left(n_{\rho}+\frac{1}{2}\right) \gamma-\frac{1}{2 n_{z}^{2}}
$$

with $n_{z}$ and $n_{\rho}$ integers $\left(n_{z}>0\right)$.

The various $n_{z}$ Rydberg series associated with different $n_{\rho}$ values are coupled by the nonadiabatic term $\frac{1}{|z|}-\frac{1}{\sqrt{\rho^{2}+z^{2}}}$. The assumption that the coupling is strong enough to completely mix the various series allows to model the full hamiltonian by a random matrix. In the situation discussed here, this assumption is simply not true. Indeed, the partial density of states in a given $n_{\rho}$ channel is $n_{z}^{3}$. As the thresholds for the various series are 
different, the corresponding $n_{z}$ values at a given total energy are also different. For example, at $E=-0.5 \gamma$, the density of states in the $n_{\rho}=0,1,2 \ldots$ series are respectively proportional to $1,2^{-1.5}, 3^{-1.5}, \ldots$ which means that there are much more energy levels of $H_{\text {sep }}$ belonging to the $n_{\rho}=0$ series than to the other series. In other words, the levels belonging to the higher $n_{\rho}=1,2 \ldots$ series are not numerous enough to destroy the regularity of the $n_{\rho}=0$ series, even when the non-adiabatic coupling is strong.

In order to model this situation, we consider a Hilbert space composed of two subspaces which we baptize "regular" and "chaotic". We construct a model hamiltonian by considering a matrix diagonal in the regular subspace with equally spaced eigenvalues (this represents the $n_{\rho}=0$ series discussed above). In the chaotic subspace (which represents the strongly mixed $n_{\rho}=1,2 \ldots$ series), we model the hamiltonian by a random matrix. The coupling of the regular states to the chaotic ones is taken constant throughout the regular series [8]. Indeed, in the physical system considered here, the Rydberg states of the $n_{\rho}=0$ series are coupled to the other series close to the nucleus, where all Rydberg states have almost identical wavefunctions.

In the limit of large matrices, this model has only two parameters: the relative weights of chaotic and regular states (i.e. the ratio of the dimensions of the chaotic and regular subspaces) and the strength of the coupling. More refined models could be used - for example taking a nonuniform density of regular states to represent a Rydberg series - but at the price of adding new parameters and losing the simplicity of the model.

We have not been able to deduce analytically the NNS distribution for our model and we suspect that it is a very difficult task. Thus, we have determined it numerically. It is shown in Figs. 11 and 2 in comparison with the NNS distribution for the hydrogen atom in a magnetic field. The agreement is obviously excellent. Especially, two important features are well reproduced: first, the absence of large level spacings. The largest observed spacing precisely corresponds to the spacing between two unperturbed states of the regular series. Hence, the largest observed spacing fixes in our model the ratio of regular to chaotic states. As expected from our interpretation, this ratio increases from Fig. 11 (ratio=1.35) to Fig. 2 
(ratio $=1.72$ ), in agreement with the increasing density of states in the $n_{\rho}=0$ channel. This leaves us with a single parameter, the strength of the regular-chaotic coupling, which is adjusted to obtain the best agreement. The differences between the predictions of the model and the numerical results on the physical system are of the order of the statistical fluctuations. This makes us confident that the model catches the essential part of the physics involved.

In order to check the validity of the model, we numerically studied the energy spectrum of a similar system, a quartic oscillator with hamiltonian:

$$
H=\frac{p_{x}^{2}+p_{y}^{2}}{2}+\frac{x^{2} y^{2}}{2}
$$

The classical motion is chaotic [2,9-11]. Although the particle can escape classically along $x$ or $y$ axis whatever its positive energy, it has been proven [2] that the quantum energy spectrum is purely discrete.

The hamiltonian (5) has a $C_{4 v}$ symmetry, leading to four non-degenerate series of energy levels, which we label EEE, EEO, OOE and OOO according to Ref. [10] (E means even, O means odd, the first two letters refer to the $x \rightarrow-x$ and $y \rightarrow-y$ symmetries, the third letter to the $x \leftrightarrow y$ symmetry), plus a twofold degenerate series (EO and OE) that we will not consider here.

The energy levels are computed through diagonalization of the hamiltonian in a harmonic oscillator basis. Matrices of size up to 922000 are used to produce the 4 independent sets of energy levels. The spectra are unfolded [11,12 and the NNS distributions computed. We find that the EEE and EEO series have similar statistical properties, as well as the OOE and OOO series. We thus collect the corresponding data in two sets. The cumulative NNS distributions are shown in Figs. 3 and 4 , together with the best fits using our regularchaotic model. Again, the agreement is very good in both cases. The phenomena already observed for the hydrogen atom in magnetic field, namely the lack of large spacings and strong deviations from the Wigner distribution, are also observed here.

As soon as the particle escapes either in the $x$ or the $y$ direction, an adiabatic separation in 
coordinates $x$ and $y$ is possible. For example, in the $x$ direction, the particle oscillates rapidly around the $y=0$ equilibrium position while moving slowly along $x$. The energy spectrum is thus composed of interacting series, each series corresponding to a well defined $n_{y}$ quantum number of the harmonic oscillator along $y$ and various states along the $x$ direction. For even- $y$ parity, the series $n_{y}=0$ has a much higher density of states than the other series $n_{y}=2,4, \ldots$, leading to strong deviations from the Wigner NNS distribution (see Fig. (3)). For odd $y$-parity, the coupled series are $n_{y}=1,3,5 \ldots$ The $n_{y}=1$ series is dominant over the other ones, but the effect is less pronounced (see Fig. 田): indeed, the $n_{y}=1$ wavefunction vanishes on the $y=0$ axis and cannot "explore" the motion along $x$ as much as the $n_{y}=0$ state. We have also checked that the numerically obtained distributions do not depend on the energy, which means that it should persist for highly excited states in the semiclassical limit.

Finally, we want to stress that the observed deviations have an intrinsic quantum origin. It is because few series interact - one being dominant - that Wigner distribution is not observed. For the hydrogen atom in a magnetic field, $E / \gamma$ has to be kept close to 0 . This means that, at constant scaled energy $\epsilon=E \gamma^{-2 / 3}$ in the semiclassical limit $E \rightarrow 0, \gamma \rightarrow 0$, the ratio $E / \gamma$ tends to $-\infty$ and the Wigner distribution is recovered. When speaking about "semiclassical limit", one has to be careful, indicating precisely how the various quantities tend to 0 .

Although the situation discussed in this letter seems to be exceptional, it has a tremendous practical importance, especially in atomic physics. Indeed, the Coulomb interaction has an infinite range and may easily produce such situations where an adiabatic separation is possible in some part of phase space. Let us give two simple examples. For the hydrogen atom in a magnetic field close to the ionization threshold, either below or above, one is always close to a Landau threshold $\left(n_{\rho}+1 / 2\right) \gamma$ where a Rydberg series converge to. Hence, strong deviations from random matrix predictions are expected everywhere in this region, either for bound states or resonances [13]. This has in fact been observed experimentally [4]. These authors discovered windows where one Rydberg series appear to be accidentally 
decoupled from the other ones, giving rise to a locally regular series. Although this decoupling is exceptional throughout the spectra, our statistical observations rely on the same phenomenon.

The second example is even more spectacular, and probably quite obvious for most atomic physicists. The helium atom is a mainly chaotic three-body system. Nevertheless, below every single ionization threshold, one observes regular Rydberg series whose NNS distribution is far from Wigner (it is actually a $\delta$-peak at $s=1$ ). This is just the extreme limit of our model when one series has a much larger density of states than all the other ones (associated with perturbers in the Rydberg series) and the chaotic subspace almost disappears.

J.Z. acknowledges partial support of KBN, grant P302 102 06. Laboratoire KastlerBrossel, de l'Ecole Normale Supérieure et de l'Université Pierre et Marie Curie, is Unité Associée 18 du Centre National de la Recherche Scientifique. 


\section{REFERENCES}

[1] O. Bohigas, M.J. Giannoni and C. Schmit, Phys. Rev. Lett. 52, 1 (1984).

[2] O. Bohigas in Chaos and quantum physics, edited by M.-J. Giannoni, A. Voros and J. Zinn-Justin, Les Houches Summer School, Session LII (North-Holland, Amsterdam, 1991).

[3] F. Haake, Quantum Signatures of Chaos (Springer, Berlin 1991).

[4] C. Iu, G.R. Welch, M.M. Kash, K. Hsu, and D. Kleppner, Phys. Rev. Lett. 63, 1133 (1989).

[5] D. Delande and J.C. Gay, Phys. Rev. Lett., 57, 2006 (1986); D. Wintgen and H. Friedrich, Phys. Rev. Lett., 57, 571 (1986); G. Wunner, U. Woelk, I. Zech, G. Zeller, T. Ertl, P. Geyer, W. Schweizer and P. Ruder, Phys. Rev. Lett., 57, 3261 (1986);

[6] H. Friedrich and D. Wintgen, Phys. Rep. 183, 37 (1989).

[7] D. Delande, A. Bommier, J.C. Gay, Phys. Rev. Lett. 66, 141 (1991).

[8] As the hamiltonian in the chaotic subspace is modelled by a GOE matrix, all states of the chaotic subspace play equivalent role (the ensemble is invariant under any orthogonal transformation). Thus the repartition of the regular-chaotic coupling over the chaotic states does not matter.

[9] B. Eckhardt, G. Hose and E. Pollak, Phys. Rev. A39 (1989) 3776.

[10] C. C. Martens, R. L. Waterland, and W. P. Reinhardt, J. Chem. Phys. 90 (1989) 2328.

[11] S. Tomsovic, J. Phys. A 24, L773 (1991).

[12] D. Delande and J. Zakrzewski, to be published.

[13] K. Dupret, D. Delande and J. Zakrzewski, to be published. 


\section{FIGURES}

FIG. 1. Cumulative nearest neighbor spacing (NNS) distribution for the hydrogen atom in a magnetic field close to the ionization threshold. The NNS distribution itself in shown in the inset. The data are collected for 3271 spacings in the energy band $[-0.4 \gamma ;-0.3 \gamma]$ for 478 values of $\gamma$. It deviates noticeably from the Wigner prediction, Eq. (1) shown as a dotted line. The dashed line represents the prediction of a simple model where a series of equally spaced levels is coupled to a GOE matrix (ratio of regular levels to chaotic levels $=1.35$ ).

FIG. 2. Same as Fig. 1, but for states in the energy band $[-0.2 \gamma ;-0.1 \gamma]$. The sample of 4293 spacings is divided in two independent samples (differing by a factor 2.5 in the average value of $\gamma$ ). The two corresponding solid lines almost coincide, which proves that the deviation from the Wigner distribution persists in the $\gamma \rightarrow 0$ limit where higher and higher excited states are considered. The ratio of regular levels to chaotic levels in the model is 1.72 .

FIG. 3. Cumulative nearest neighbor spacing distribution for the quartic oscillator, Eq. (5). Only the two series of states symmetric with respect to the $x$ and $y$ axis are shown here. There are 381 level spacings included (after removing the first 100 levels from each subset; the number of removed states does not change the shape of the distribution). The dashed line represents the prediction of our regular-chaotic model (ratio of regular levels to chaotic levels $=2.64$ ). The inset shows the NNS distribution itself.

FIG. 4. Same as Fig. 3, but for the two series of states antisymmetric with respect to the $x$ and $y$ axes. There are 924 level spacings included. The dashed line represents the prediction of our regular-chaotic model (ratio of regular levels to chaotic levels $=0.82$ ). 


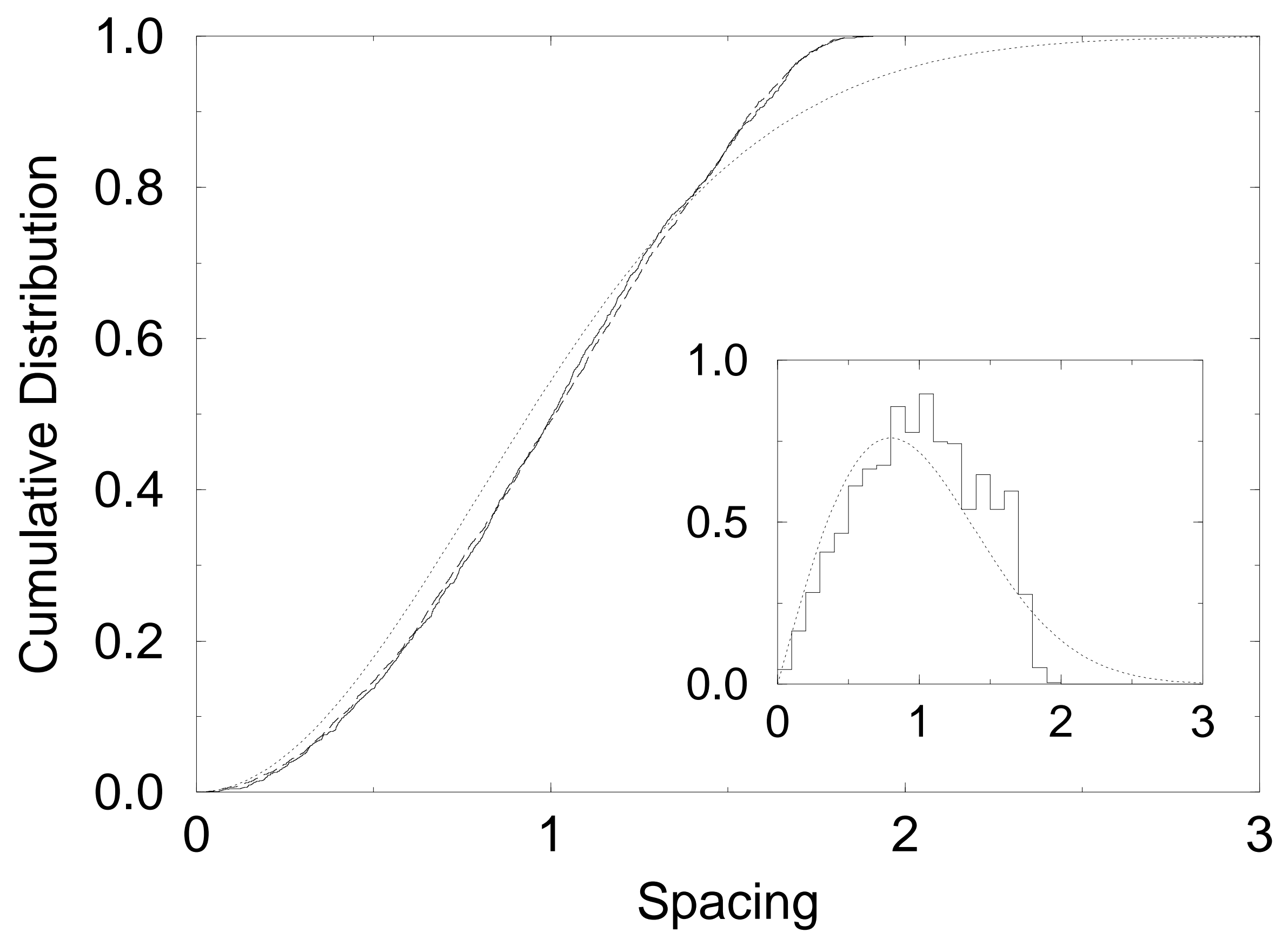




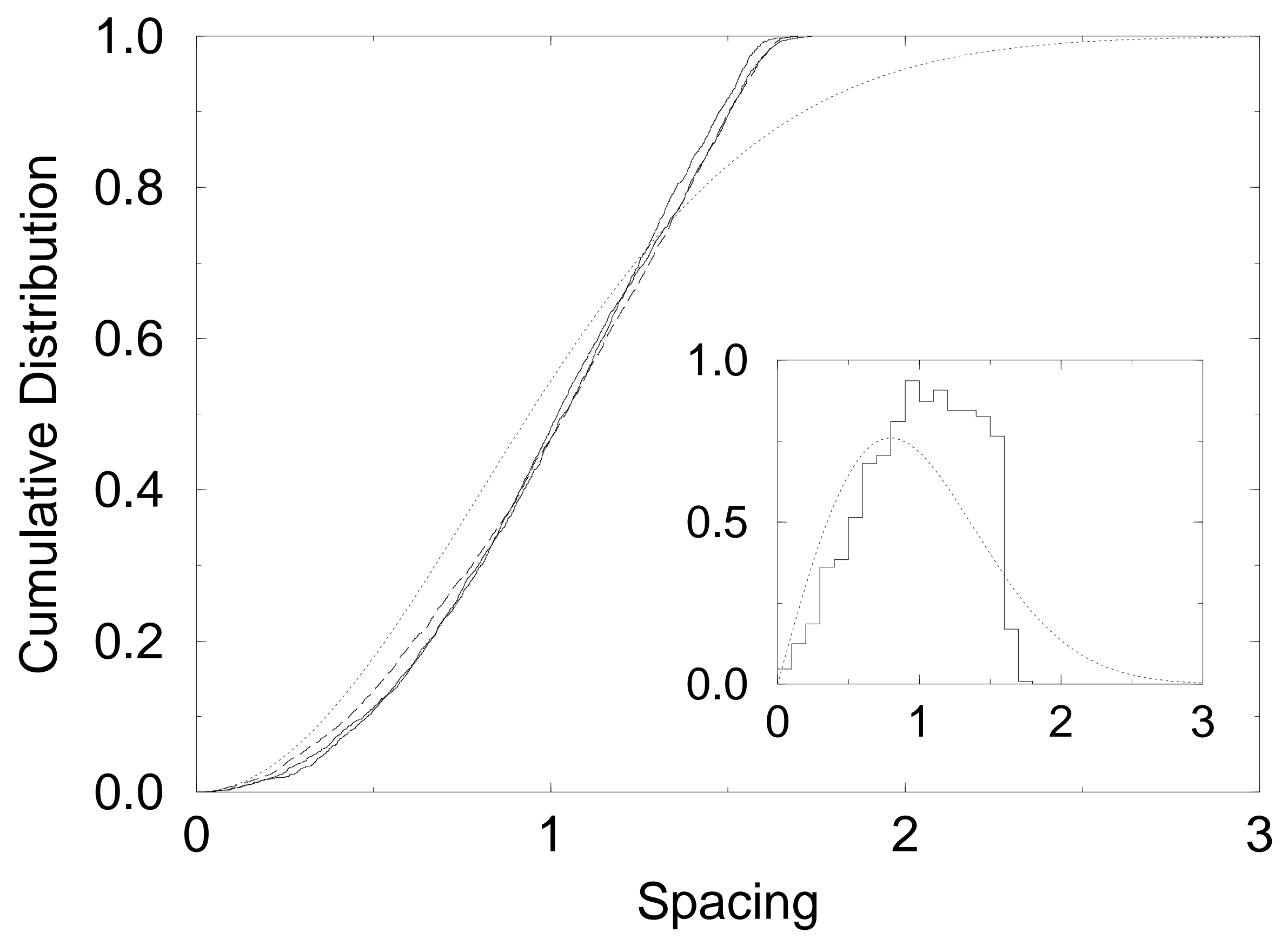




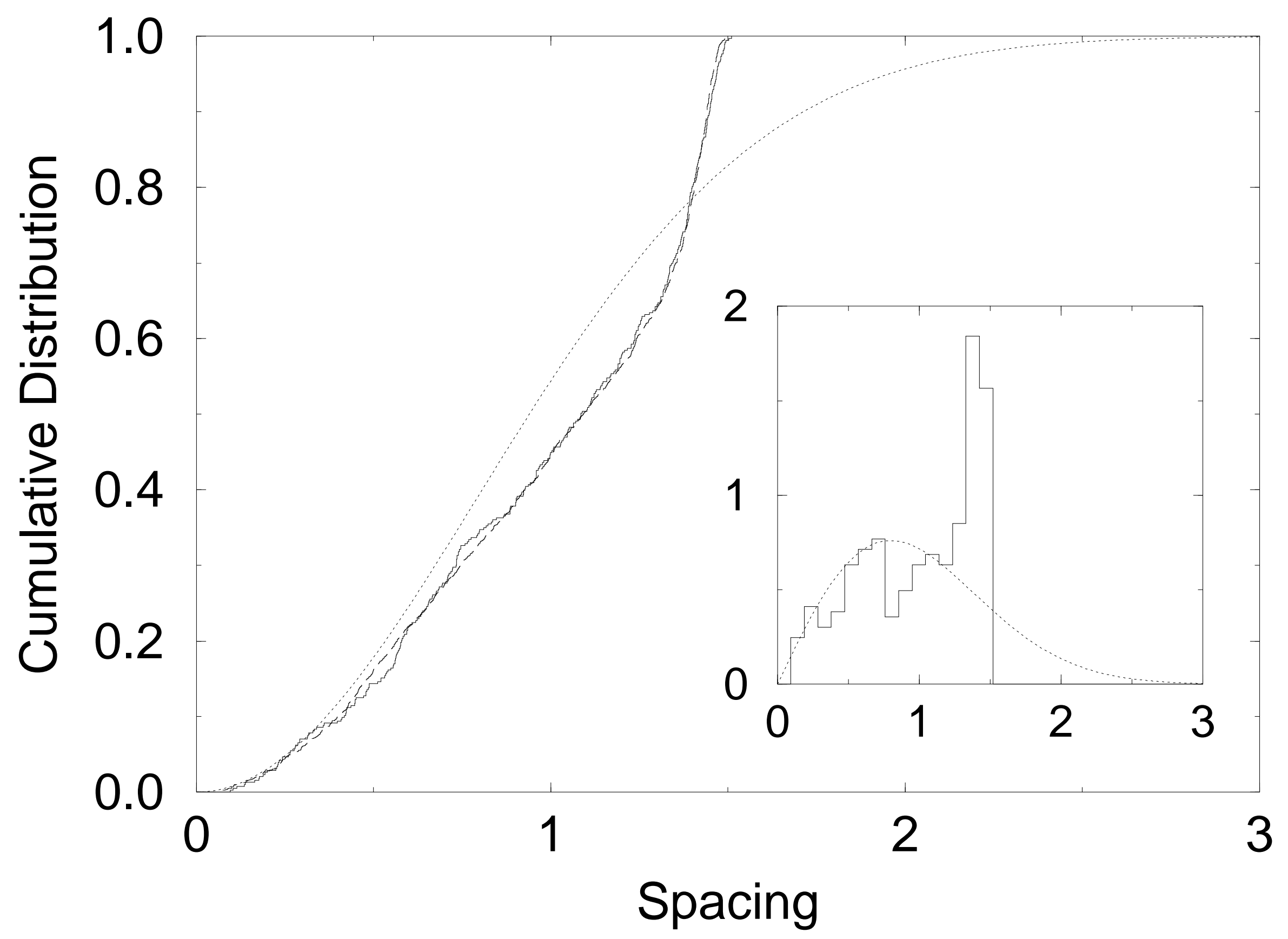




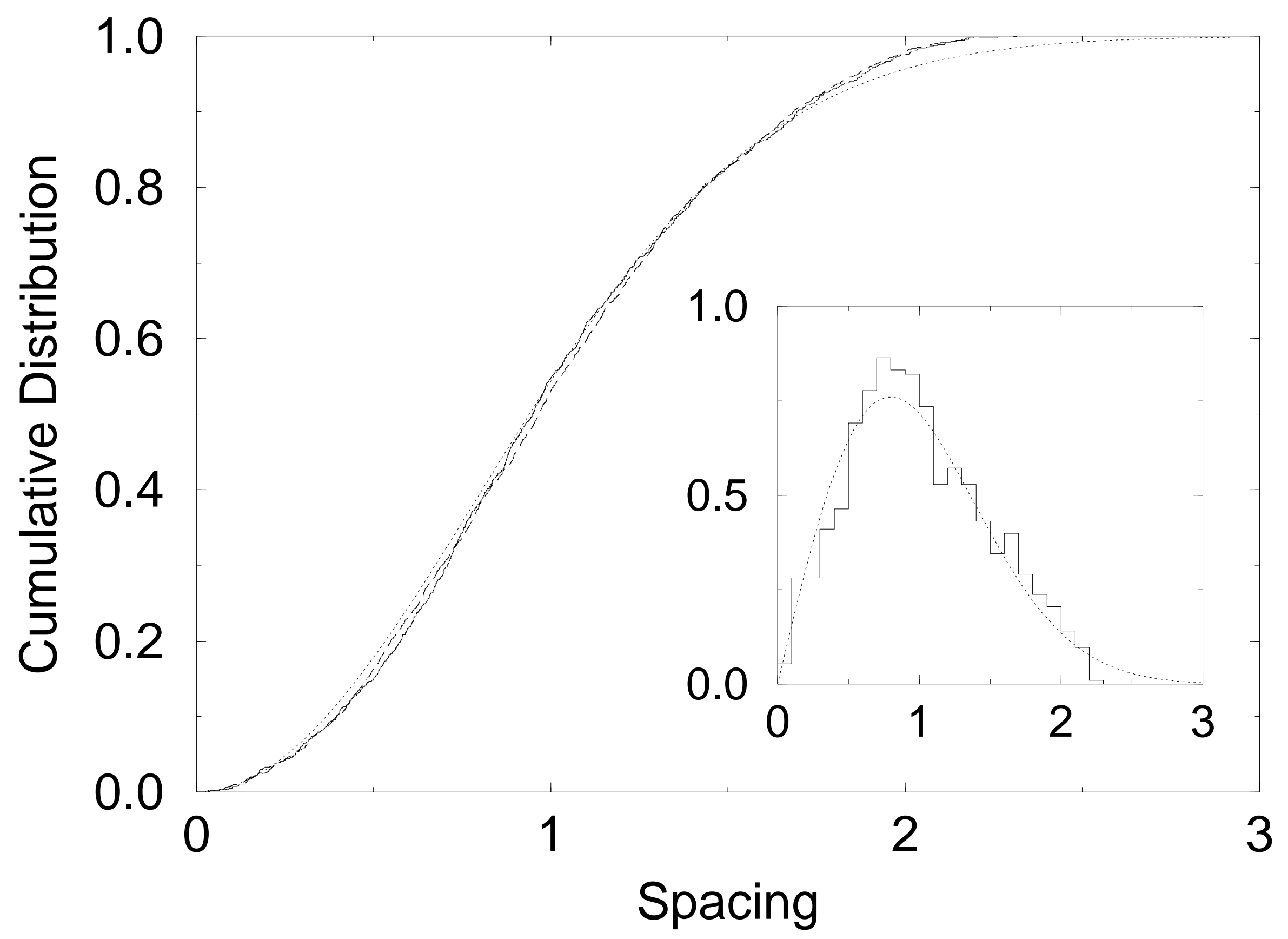

